WeLsCh, M. (1958). J. gen. Microbiol. 18, 491-497

\title{
Lysis by Agents of Microbial Origin
}

\author{
BY M. WELSCH \\ Laboratoires de Microbiologie Générale et Médicale, University of Liège
}

Instances of bacterial cultures undergoing spontaneous dissolution, and examples of micro-organisms or microbial products digesting the cells of various bacteria have often been reported since the very beginnings of bacteriology. Research in this field was undertaken from many different angles according to the fashion of the day. Early studies were mostly conducted with practical applications in view. Thus lysates were studied as vaccines. Later on, it was announced by Schiller that highly specific bacterial lysins could be obtained, almost at will, through a process called induced or forced antagonism, and that such lysins had therapeutic virtues. This idea emerged again and again during the ensuing years, but was never satisfactorily proved. Bacteriolysis was also used as a tool in order to obtain, free from cells, components with antigenic, toxic, enzymic or other properties.

The biological significance of bacteriolysis did not escape attention. Its role in Nature, as a factor involved in the cycle of the biogenic elements, was pointed out early by A. Gratia and later on by others. Hints can also be found in the literature of a possible relation between autolysis, on the one hand, and spore formation and germination on the other hand (Halvorson \& Church, 1957), as well as between autolysis and the release of exotoxins (Boroff, 1955). The importance of the lytic process in bacteriophage infection has long been recognized, but its study was rather overshadowed by that of the bacterial virus itself. Lastly, the mechanism of bacteriolysis attracted the attention of scientists. In this connexion, the possible role of the Gram-positive substrate was first stressed. More recently, and mainly following Salton's work on lysozyme (Salton, 1957), emphasis was laid on the role of the cell wall (Work, 1957).

It thus appears that the subject of the present report is indeed a very broad one. As it would be impossible to cover all its aspects, a few topics will be selected and briefly considered, the reader being referred to a recent review (Welsch, 1957) for more details and additional references. The main purpose of this paper will be to show that a great number of received ideas will have to be revised in the light of modern knowledge of bacterial anatomy.

The most obvious manifestation of bacteriolysis--what indeed should only be called lysis in the strictest sense of the word-is the progressive clearing of an initially turbid bacterial suspension. This may occur without the help of any exogenous agent: it is then called autolysis. On the contrary, it may be promoted by an extraneous agent and can then be described as heterolysis. The exogenous agent may be mechanical, physical, chemical or biological. We are concerned here with the latter case, and only with the particular instance 
when the agent promoting lysis has a microbial origin. Two different situations are to be considered: either the microbial agent at play, having a truly and directly bacteriolytic action, dissolves the cell or part of it, or it simply modifies the bacteria submitted to its action in such a way that they undergo autolysis. An agent of the latter type can be called an inducer of autolysis. It follows that an examination of the autolytic process is a pre-requisite to the study of heterolysis.

However, it can be no simple matter to decide, in a given instance, whether one deals with a truly bacteriolytic agent or with a mere inducer of autolysis. A valid criterion allowing the choice between these two hypotheses would be the possibility of using a highly specific inhibitor of the autolytic system. Indeed, if such an inhibitor did prevent heterolysis, one might safely conclude that the phenomenon observed was induced autolysis. Unfortunately we have no means of inhibiting autolysis without simultaneously more or less injuring the bacteria. In fact, inhibition of the autolytic system is most often obtained by heating, a treatment which is likely to alter the outer bacterial structures and to thus expose cell substrates which are otherwise protected. Also, heat denaturation can make sensitive to the lytic enzymes parts of the cell that are normally resistant, and conversely. Lastly, cell components are likely to diffuse from the cell during heating. Examples of such situations are known, and the use of heated bacteria has been criticized by Salton. Keeping these important reservations in mind, I believe, nevertheless, that study of the bacteriolysis of killed bacteria can give useful information. In particular, it should be stressed that microbial enzymes able to dissolve heated bacteria (e.g. actinozyme) do not act merely as the usual proteases of the digestive juices but can display a definite specificity for their bacterial substrate.

It is generally taken for granted that autolysis is an enzymic phenomenon ascribable to the action of a number of different enzymes, making, all together, what is often called the autolytic system. According to Dubos, the infra-microscopic organization of the living cell prevents the hydrolytic enzymes from digesting the cell components. When, however, the intracellular heterogeneity is not maintained for any reason whatsoever, enzymes and potential substrates are mixed together, the former digesting the latter and autolysis ensuing. Energy being necessary to maintain the cellular infra-structure, it follows that any agent, endogenous or exogenous, which interferes with energy supply or utilization may induce the process of autolysis. Of course, the autolytic systems of different bacteria are more or less potent and stable and this explains their greater or smaller liability to undergo lysis. In keeping with modern observations, it may also be supposed that bacterial species which are particularly resistant to autolysis are so because they lack an enzyme capable of destroying their cell wall and of thus promoting their ultimate disintegration.

If autolysis of a cell is a strictly endogenous process, autolysis of a bacterial population, on the contrary, is both endogenous and exogenous, since the autolytic system liberated from lysed individuals can conceivably act upon other members of the population from the outside. Such is the case, as we have shown, in autolysis of staphylococci. The autolytic system of this organism is 
released through autolysis and also, in suitable conditions, by growing colonies, provided that a minimal amount of phosphate is present. It dissolves not only the homologous and other strains of staphylococci, but also Micrococcus lysodeikticus, Sarcina lutea and, to a certain extent, Escherichia coli and Proteus vulgaris. It is certainly a complex mixture of enzymes, among which a lysozyme-like agent might be present (Mitchell \& Moyle, 1957).

When liberated from the cells and used as an agent to promote heterolysis, the autolytic system is frequently called an autolysin. It does not follow, however, that an active autolysin is necessarily produced whenever autolysis occurred. Thus lysates of Escherichia coli and Salmonella spp., obtained through the action of pyrophosphate or glycine or both, have no appreciable lytic activity. These two agents appear to act as inducers of autolysis and exert a synergistic effect, a fact which suggests that induction of autolysis may be realized in more than one way. Electrophoretic and serological studies also show that the products of lysis are different when different inducers are used (Salmon, 1957).

This picture of autolysis, natural or induced, was widely accepted, since it gave a coherent and reasonable explanation (although in rather vague terms) of many cases of autolysis, of the bacteriolytic activities shown by several antiseptics and antibiotics, even of the lysis of phage-infected bacteria. A deeper analysis of some particular cases has shown however that, in some instances, bacteriolysis is not primarily an enzymic phenomenon and, in some others, that it need not involve the hydrolytic enzymes or autolytic system of the cell. Thus Newton's work on the mode of action of polymyxin has clearly and elegantly demonstrated that fixation of the antibacterial agent upon the cytoplasmic membrane alters its permeability properties and promotes the leakage of cell material. No doubt, other agents, formerly believed to be inducers of autolysis, will be found to act in this much more direct way.

Meadow \& Work (1956), Meadow, Hoare \& Work (1957) and Rhuland (1957) have reported that Escherichia coli strain 173-25, a mutant requiring $\alpha, \epsilon$-diaminopimelic acid (DAP) for growth, lyses when cultivated in the presence of limiting amounts of this substance. Lack of enough DAP seems to prevent the organism from building its cell wall, and therefore the fragile naked cells which are formed undergo a primarily osmotic lysis. This mechanism, in which again the autolytic system plays at best a secondary role, will very likely be found responsible for other cases of autolysis.

In fact, a similar explanation was recently offered to account for the bacteriolytic activity of penicillin upon growing cells, a phenomenon which was formerly regarded by many as the result of an activation of the autolytic system. Following Lederberg's observation $(1956,1957)$ that Escherichia coli rods submitted to the action of the antibiotic in a hypertonic sucrose medium are transformed into large osmotically fragile bodies resembling protoplasts, it is now believed that penicillin interferes with cell-wall synthesis. The characterization of cell-wall components in the cultures of penicillin-treated bacteria (Park, 1952 ; Park \& Strominger, 1957) is in good agreement with this interpretation. The precise point of action of the antibiotic has yet to be 
determined. It might prevent entry into the cell of a key substance necessary for cell-wall synthesis, or it might inhibit an enzyme taking part in this process. It might also disorganize the cytoplasmic membrane, a structure which is almost certainly involved in the formation of the cell wall. In fact, the permeability alterations which quickly follow upon the addition of penicillin to a bacterial suspension are rather in favour of this opinion. It is also possible that penicillin induces the production by the cell of a wall-destroying enzyme (Prestidge \& Pardee, 1957). Again, this suggests that other agents which induce bacteriolysis may act according to a similar mechanism.

The role of the autolytic system in the lysis of phage-infected bacteria can be easily accepted in the case of those organisms which are known to autolyse readily. It is less acceptable for those many species which can be phageinfected but which are generally resistant to autolysis. Thus, the more or less specific lytic activities recorded in phage lysates of staphylococci by Gratia, by Wollman, and, more recently, by Wahl \& Josse-Goichot (lyse par entraînement) or by Welsch, Cavallo \& Cantelmo (lysis in the cold and collateral lysis of ultraviolet induced lysogenic staphylococci), were ascribed mainly to the normal autolytic system. In other cases, however, it appears that abnormal lytic systems are synthesized as a result of the viral infection. They may be agents distinct from the phage or components of the virus particle.

In the first group should be mentioned the phage-lysin of Sertic \& Bulgakov, the virolysin of Ralston and collaborators, the prolysins of Huppert \& Panijel, the endolysins to be discussed in this symposium by Jacob, and the streptolytic agent recently described by Maxted (1957). Their mode of action is generally unknown. It is permissible to mention with them the capsuledestroying enzyme synthesized by a strain of Klebsiella pneumoniae upon its being infected by a certain phage (Adams \& Park, 1956; Park, 1956). It must also be recalled that procedures effective to induce lysogenic bacteria can promote the synthesis of a bacteriolytic agent (megacin) by some strains of Bacillus megaterium (Ivánovics \& Alföldi, 1957). In the second group, one finds the endogenous prolysins of Panijel \& Huppert (1957) and the wall-destroying enzyme of coliphages to be discussed later on by Weidel. From this brief review, it appears that all cases of autolysis, spontaneous or induced, cannot be explained by the activation of an autolytic system. This concept, however, should not be discarded outright since it probably still has a useful role to play.

Studying the controlled autolysis of pneumococci, Dubos observed that the first step of this process was a transformation of the organisms from the Grampositive to the Gram-negative state. The change was enzymic in nature and the 'Gram-negativating' enzyme was supposed to be the one really initiating autolysis. This idea was taken up and elaborated upon by Stacey and his school. In fact, they devised a theory, supposed to be of general application, to account for the autolysis of Gram-positive organisms. According to that theory, which was later on expanded to explain the bacteriolytic activities of a Streptomyces sp., sensitive bacteria are first changed to Gram-negative cytoskeletons through the action of a nuclease, disintegration of the cells being thereafter brought about by two or more proteases. As a result of our own 


\section{Lysis by agents of microbial origin}

observations, we have never been convinced that loss of the Gram-positive character was necessary to make the cell sensitive to the properly lytic enzymes. Neither did the loss of Gram-positiveness appear to us as the result of the destruction of a substrate specifically linked with the Gram reaction. Rather we thought it merely expressed a general loss of ribonucleic acid with concomitant decrease of basophily. It is relevant to point out here that almost all methods used to obtain Gram-negative cyto-skeletons can be used just as well -in Gram-negative as well as in Gram-positive organisms-to remove cytoplasmic nucleic acids and thus show the bacterial nuclei.

Innumerable publications have reported the ability of various microorganisms to clarify turbid bacterial suspensions. Representatives of the genera Bacillus and Streptomyces are prominent in this respect. In many of these cases, very likely, an antibiotic is produced which, acting as inducer of autolysis or promoting permeability troubles, is responsible for the lytic process. In many others, one may be dealing with truly lytic agents, but in a few instances only has the mechanism of true heterolysis been studied and elucidated.

The production of lysozyme by Micrococcus lysodeikticus (Fleming \& Allison), by Streptomyces violaceus (Kriss) and by $S$. albus (McCarty), has been reported, but without entirely satisfactory evidence. In contrast, the cell-wall dissolving enzyme obtained from a Bacillus subtilis by Richmond (1957) appears to be a true bacterial lysozyme.

A lytic agent, obtained from anaerobic autolysates of a Bacillus subtilis was obtained as a concentrated, but still far from pure, preparation by Nomura \& Hosoda $(1956 a, b)$. According to its spectrum of activity, it is unlikely to be identical with lysozyme. It acts upon the cell walls of $B$. subtilis and $B$. megaterium, but has no action on B. cereus, Micrococcus lysodeikticus, Sarcina lutea, Staphylococcus aureus or Escherichia coli. However, it liberates reducing groups from its substrates and is considered as a polysaccharidase.

A cell-wall dissolving enzyme was recognized by Strange \& Dark (1957) in the aqueous extracts of spores from Bacillus cereus and avirulent strains of $B$. anthracis. It acts upon the vegetative cell walls and spore coats of several bacilli. Unlike lysozyme, it is more active upon $B$. cereus than upon $B$. megaterium cell walls. It liberates from its substrates non-dialysable fragments containing all the 'basal' components of the bacterial cell wall. It should not be hastily concluded that the bacteriolytic activities of all Bacillus spp. are due to specific cell-wall digestion. In fact, Norris (1957) described the lysis of several Bacillus spp., mainly in the presence of thiomersalate, by a culturefiltrate from a $B$. cereus. Under its action, the sensitive organisms first lost their Gram-positive character, and then their capacity to retain the counterstain; but the cell wall could still be demonstrated by appropriate staining procedures.

The bacteriolytic properties of streptomycetes have been known since the early observations of Lieske and of Gratia \& Dath. Their active filtrates (actinomycetin) are able to dissolve the suspensions of killed Gram-negative organisms and of some intact Gram-positive bacteria. The protein nature of 
the active principle(s) and the enzymic nature of the lytic process were demonstrated. It was believed, originally, that the agent acting upon heat-killed Escherichia coli (actinozyme) was involved, together with some additional principle, in the lysis of intact Gram-positive bacteria. This idea was also developed by Webb and his co-workers and more recently by Klinge (1955). However, Ghuysen has clearly shown that actinozyme and the staphylolytic system are distinct. They appear in, and disappear from, the culture medium at different moments. The optimal conditions for their production and for their activity, respectively, are different. They are adsorbed on, and eluted from, various substrates according to different physico-chemical conditions. From the culture-filtrates of a Streptomyces albus, McCarty isolated a streptolytic enzyme which was shown, by him and by Salton, to dissolve the cell wall of the sensitive organisms and was identified as a polysaccharidase distinct from lysozyme. Following this observation, our actinomycetin was found to contain, in addition to actinozyme, the staphylolytic system and several proteases, a streptolytic agent, possibly identical with McCarty's, and at least two pneumolytic principles. The staphylolytic system was isolated by Ghuysen and found to comprise two components: F 1 and F2. The F1 component was obtained as a highly purified protein. Ghuysen and Salton studied the bacteriolytic spectra of these two agents. They are somewhat different, at least quantitatively. F1 and F2 act synergistically and their bacteriolytic activity is completely parallel to their digesting action upon the corresponding cell walls. They are different from lysozyme and from McCarty's enzyme, since they liberate from their substrates no reducing groups, no sugars, no amino sugars, but only unidentified small peptides, alanine and/or glycine, together with a large amount of undialysable material. They are regarded as peptidases and, to recall their origin as well as their bacteriolytic properties, they have been named actinolysopeptidases.

In conclusion, it can be stated that the few truly bacteriolytic agents as yet obtained in a purified state, act in destroying the cell wall of the sensitive cell, by degradation of either its polysaccharidic or its protein moiety.

\section{REFERENCES}

Adams, M. H. \& Park, B. H. (1956). An enzyme produced by a phage-host cell system. II. The properties of the polysaccharide depolymerase. Virology, 2, 719.

Bonoff, D. A. (1955). Studies of toxins of Clostridium botulinum. III. Relation of autolysis to toxin production. J. Bact. 70, 363.

Halvorson, H. \& Church, B. (1957). Biochemistry of spores of aerobic bacilli with special reference to germination. Bact. Rev. 21, 112.

IvÁNovics, G. \& ALFöLDI, L. (1957). Bacteriocinogenesis in Bacillus megaterium. J. gen. Microbiol. 16, 522.

Kuinge, K. (1955). Das extrazelluläre bakteriolytische Fermentsystem der Streptomyceten. Arch. Hyg. 139, 565.

Lederberg, J. (1956). Bacterial protoplasts induced by penicillin. Proc. nat. Acad. Sci., Wash. 42, 574.

Lederberg, J. (1957). Mechanism of action of penicillin. J. Bact. 73, 144.

Maxted, W. R. (1957). The active agent in nascent phage lysis of streptococci. J. gen. Microbiol. 16, 584. 
Meadow, P., Hoare, D. S. \& Work, E. (1957). Interrelationships between lysine and $\alpha, \epsilon$-diaminopimelic acid and their derivatives and analogues in mutants of Escherichia coli. Biochem. J. 66, 270.

Meadow, P. \& Work, E. (1956). Interrelationships between diaminopimelic acid, lysine and their analogues in mutants of Escherichia coli. Biochem. J. 64, $11 \mathrm{P}$.

Mitchell, P. \& Moyle, J. (1957). Autolytic release and osmotic properties of 'protoplasts' from Staphylococcus aureus. J. gen. Microbiol. 16, 184.

Nomura, M. \& Hosoda, J. (1956a). Action of an autolytic enzyme of Bacillus subtilis on the cell wall. Nature, Lond. 177, 1037.

Nomura, M. \& Hosoda, J. $(1956 b)$. Nature of the primary action of the autolysin of Bacillus subtilis. J. Bact. 72, 573.

Norris, J. R. (1957). A bacteriolytic principle associated with cultures of Bacillus cereus. J. gen. Microbiol. 16, 1.

Panijel, J. \& Huppert, J. (1957). Mise en évidence de prolysines endogènes dans les bactériophages. Ann. Inst. Pasteur, 93, 352.

Park, B. H. (1956). An enzyme produced by a phage-host cell system. I. The properties of a Klebsiella phage. Virology, $2,711$.

Park, J. T. (1952). Uridine-5'-pyrophosphate derivatives. J. biol. Chem. 194, 877, $885,897$.

Park, J. T. \& Strominger, J. L. (1957). Mode of action of penicillin. Science, 125, 99.

Prestidge, L. S. \& Pardee, A. B. (1957). Induction of bacterial lysis by penicillin. J. Bact. 74, 48.

Rhuland, L. E. (1957). Role of $\alpha, \epsilon$-diaminopimelic acid in the cellular integrity of Escherichia coli. J. Bact. 73, 778.

Richmond, M. H. (1957). Bacterial lysozyme. J. gen. Microbiol. 16, iv.

Salmon, J. (1957). Constitution antigénique des lysats de Salmonella schottmuelleri. Rev. belge Path. (in the Press).

Salton, M. R. J. (1957). The properties of lysozyme and its action on microorganisms. Bact. Rev. 21, 82.

Strange, R. E. \& Dark, F. A. (1957). A cell-wall lytic enzyme associated with spores of Bacillus species. J. gen. Microbiol. 16, 236.

Welsch, M. (1957). Activités bactériolytiques des microorganismes. Ergebn. Mikrobiol. Immunit. 30, 217.

Work, E. (1957). Biochemistry of the bacterial cell wall. Nature, Lond. 179, 841. 